J. Korean Math. Soc. 48 (2011), No. 4, pp. 669-689

DOI 10.4134/JKMS.2011.48.4.669

\title{
ON LORENTZIAN QUASI-EINSTEIN MANIFOLDS
}

\author{
Absos Ali Shaikh ${ }^{\dagger}$, Young Ho Kim*, and Shyamal Kumar Hui
}

Abstract. The notion of quasi-Einstein manifolds arose during the study of exact solutions of the Einstein field equations as well as during considerations of quasi-umbilical hypersurfaces. For instance, the RobertsonWalker spacetimes are quasi-Einstein manifolds. The object of the present paper is to study Lorentzian quasi-Einstein manifolds. Some basic geometric properties of such a manifold are obtained. The applications of Lorentzian quasi-Einstein manifolds to the general relativity and cosmology are investigated. Theories of gravitational collapse and models of $\mathrm{Su}-$ pernova explosions [5] are based on a relativistic fluid model for the star. In the theories of galaxy formation, relativistic fluid models have been used in order to describe the evolution of perturbations of the baryon and radiation components of the cosmic medium [32]. Theories of the structure and stability of neutron stars assume that the medium can be treated as a relativistic perfectly conducting magneto fluid. Theories of relativistic stars (which would be models for supermassive stars) are also based on relativistic fluid models. The problem of accretion onto a neutron star or a black hole is usually set in the framework of relativistic fluid models. Among others it is shown that a quasi-Einstein spacetime represents perfect fluid spacetime model in cosmology and consequently such a spacetime determines the final phase in the evolution of the universe. Finally the existence of such manifolds is ensured by several examples constructed from various well known geometric structures.

\section{Introduction}

It is well known that a semi-Riemannian manifold $\left(M^{n}, g\right), n \geq 3$, is Einstein if its Ricci tensor $S$ of type $(0,2)$ is of the form $S=\alpha g$, where $\alpha$ is a constant, which reduces to $S=\frac{\kappa}{n} g, \kappa$ being the scalar curvature (constant) of the manifold. Let $\left(M^{n}, g\right), n \geq 3$, be a semi-Riemannian manifold. Let

Received November 11, 2008.

2010 Mathematics Subject Classification. 53B30, 53B50, 53C50, 53C80, 83D05.

Key words and phrases. quasi-Einstein manifold, Lorentzian manifold, Lorentzian quasiEinstein manifold, Lorentzian quasi-constant curvature, conformally flat, scalar curvature, Codazzi tensor, perfect fluid spacetime, viscous fluid spacetime, heat flux, concircular structure spacetime.

$\dagger$ Partially supported by grant from CSIR, New Delhi, India (Project F. No. 25(0171)/09/EMR-II).

* Partially supported by grant from KOSEF, R01-2007-000-20014-0.

(C)2011 The Korean Mathematical Society 
$U_{S}=\left\{x \in M: S \neq \frac{\kappa}{n} g\right.$ at $\left.x\right\}$. Then the manifold $\left(M^{n}, g\right)$ is said to be quasiEinstein manifold ([7], [10], [11], [12], [13], [14], [17], [18], [19], [21], [22]) if on $U_{S} \subset M$, we have

$$
S-\alpha g=\beta A \otimes A,
$$

where $A$ is an 1 -form on $U_{S}$ and $\alpha, \beta$ are some functions on $U_{S}$. It is clear that the 1-form $A$ as well as the function $\beta$ are non-zero at every point on $U_{S}$. From the above definition it follows that every Einstein manifold is quasi-Einstein. In particular, every Ricci-flat (e.g. Schwarzschild spacetime) manifold is quasiEinstein. The scalars $\alpha, \beta$ are known as the associated scalars of the manifold. Also the 1-form $A$ is called the associated 1-form of the manifold defined by $g(X, \rho)=A(X)$ for any vector field $X ; \rho$ being a unit vector field, called the generator of the manifold. Such an $n$-dimensional quasi-Einstein manifold is denoted by $Q E_{n}$.

Einstein manifolds form a natural subclass of the class of quasi-Einstein manifolds. Another subclass of quasi-Einstein manifolds is Ricci simple manifolds, which are semi-Riemannian manifolds having the Ricci tensor of rank at most 1 ([8]). In [15] Deszcz et al. proved that every three dimensional quasiEinstein manifold is pseudo symmetric and conversely. Also three dimensional Cartan hypersurfaces are quasi-Einstein manifolds ([16]). In [10] Deszcz et al. studied 3-dimensional quasi-Einstein totally real submanifolds in 6-dimensional sphere. Again it may be mentioned that every quasi-umbilical hypersurface $M$ in a semi-Riemannian space of constant curvature $N^{n+1}(c), n \geq 4$, is a quasiEinstein manifold ([13], p. 376).

An $n$-dimensional Lorentzian manifold $M$ is a smooth connected paracompact Hausdorff manifold with a Lorentzian metric $g$, that is, $M$ admits a smooth symmetric tensor field $g$ of type $(0,2)$ such that for each point $p \in M$, the tensor $g_{p}: T_{p} M \times T_{p} M \rightarrow \mathbb{R}$ is a non-degenerate inner product of signature $(-,+, \ldots,+)$, where $T_{p} M$ denotes the tangent vector space of $M$ at $p$ and $\mathbb{R}$ is the real number space. A non-zero vector $v \in T_{p} M$ is said to be timelike (resp. non-spacelike, null, spacelike) if it satisfies $g_{p}(v, v)<0$ (resp. $\leq 0,=0,>0)([3],[31])$.

The present paper deals with a study of Lorentzian quasi-Einstein manifolds. A Lorentzian quasi-Einstein manifold (briefly, $L Q E_{n}$ ) is a quasi-Einstein manifold with the generator $\rho$ as the unit timelike vector field such that $g(\rho, \rho)=-1$. Thus a $L Q E_{n}$ is a Lorentzian manifold whose Ricci tensor satisfies (1.1) such that the generator $\rho$ is the unit timelike vector field. Hence, a $Q E_{n}$ with a Lorentzian metric is a $L Q E_{n}$.

Section 2 is concerned with some basic geometric properties of $L Q E_{n}$ and it is shown that in such a manifold, the generator $\rho$ is generic. Then we obtain a sufficient condition for a Lorentzian manifold to be of $L Q E_{n}$. In an Einstein manifold the scalar curvature is always constant. But in a $L Q E_{n}$ it is not true in general. Then we obtain a sufficient condition for the scalar curvature of a $L Q E_{n}$ to be a constant. If the scalar curvature of a $L Q E_{n}$ is constant, then 
its associated scalars are not constants, in general. However, if the associated scalars are constants, then it is a manifold of constant scalar curvature. It is shown that in a Ricci symmetric $L Q E_{n}$, the associated scalars are constants and the associated 1-form $A$ is closed. Also it is proved that if the Ricci tensor of a $L Q E_{n}$ is of Codazzi type and the associated 1-form $A$ is closed, then the generator of the manifold and the vector field $\operatorname{grad} \alpha-\operatorname{grad} \beta$ are co-directional.

Section 3 deals with the conformally flat $L Q E_{n}$. It is proved that a conformally flat $L Q E_{n}$ is a manifold of Lorentzian quasi-constant curvature. Every manifold of Lorentzian quasi-constant curvature is a $L Q E_{n}$ but the converse is not true, in general. However a 3-dimensional Lorentzian quasi-Einstein manifold is of Lorentzian quasi-constant curvature. The notion of quasi-constant curvature, introduced by Chen and Yano ([4]), is an important geometric notion as the manifold of quasi-constant curvature is a natural subclass of quasiEinstein manifolds. Also from the investigation of Karchar ([25]), it follows that a conformally flat perfect fluid spacetime has the geometric structure of quasi-constant curvature. It may be noted that the sectional curvature of a conformally flat $L Q E_{n}$ is non-vanishing.

In Section 4 we investigate the applications of $L Q E_{n}$ to the general relativity and cosmology. A spacetime is a 4-dimensional connected Lorentzian manifold. Hence a quasi-Einstein spacetime is a connected $L Q E_{4}$. In general relativity the matter content of the spacetime is described by the energy momentum tensor $T$ which is to be determined from physical considerations dealing with the distribution of matter and energy. Since the matter content of the universe is assumed to behave like a perfect fluid in the standard cosmological models, the physical motivation for studying Lorentzian manifolds is the assumption that a gravitational field may be effectively modeled by some Lorentzian metric defined on a suitable four dimensional manifold $M$. The Einstein equations are fundamental in the construction of cosmological models which imply that the matter determines the geometry of the spacetime and conversely the motion of matter is determined by the metric tensor of the space which is non-flat.

The "singularity theorems" deduced by Stephen Hawking and Roger Penrose play the crucial role in general relativity. The theorems assert that any "reasonable" model of the universe in which we live must be "singular", that is contain regions in which the laws of physics as we know them must break down. According to Penrose the singularities arise from gravitational collapse (black holes), whereas Hawking are concerned with the existence of cosmological singularities (the big bang). Relativistic fluid models are of considerable interest in several areas of astrophysics, plasma physics and nuclear physics. In most models a key feature is the occurrence of an outward propagating relativistic shock. The effects of deviations from spherical symmetry due to an initial angular momentum and magnetic field must also be assessed, which requires the use of relativistic magneto fluid dynamical models. Several theories of Jets and Superluminal variations in extra galactic radio sources and Quasars 
are based on relativistic magneto-fluid models. In particular, in some models, the observed variability is associated with the development of magneto-fluid dynamical waves or instabilities. In the field of plasma physics there are areas where relativistic fluid models are of interest. Intense relativistic electron beams, which have very interesting applications such as the free electron laser, have also been modeled as relativistic fluids near thermal equilibrium. In situations far from thermal equilibrium, relativistic fluid models with generalized state equations have been proposed in a non covariant framework. More satisfactory fully covariant models have also been introduced $([1])$. In the field of nuclear physics, high energy-collisions among heavy nuclei have been modeled by using relativistic fluid dynamics. When a non dissipative description applies and relativistic effects are not negligible, nuclear matter is described by the equations of relativistic fluid dynamics and all the detail of nuclear interactions are incorporated in the state equation. Many models predict the occurrence of a relativistic shock when to heavy nuclei collide. Also, some models under current investigation predict that relativistic shocks (or detonation and deflagration waves) might be related to phase transition from nuclear matter to quark-gluon plasma.

It is shown that a perfect fluid spacetime obeying Einstein's equation with a cosmological constant is a connected $L Q E_{4}$ with the generator $\rho$ as the flow vector field of the fluid. Thus a quasi-Einstein spacetime can be viewed as a model of the perfect fluid spacetime. Also it is proved that in a viscous fluid quasi-Einstein spacetime none of the isotropic pressure and energy density can be a constant and also the matter content of such a spacetime is a nonthermalised fluid. It is shown that in a viscous fluid quasi-Einstein spacetime with Codazzi type energy momentum tensor the flow vector field of the fluid is a unit proper concircular vector field and hence such a spacetime is a concircular structure spacetime ([38], [39]).

The physical motivation for studying various types of spacetime models in cosmology is to obtain the information of different phases in the evolution of the universe, which may be classified into three phases, namely, the initial phase, the intermediate phase and the final phase. The initial phase is just after the Big Bang when the effects of both viscosity and heat flux were quite pronounced. The intermediate phase is that when the effect of viscosity was no longer significant but the heat flux was still not negligible. The final phase, which extends to the present state of the universe when both the effects of viscosity and heat flux have become negligible and the matter content of the universe may be assumed to be perfect fluid. The study of $L Q E_{n}$ is important because such spacetime represents the third phase in the evolution of the universe. Consequently the investigations of quasi-Einstein manifolds helps us to have a deeper understanding of the global character of the universe including the topology, because the nature of the singularities can be defined from a differential geometric stand point.

The last section deals with some examples of $L Q E_{n}$. 


\section{Preliminaries}

We define on a semi-Riemannian manifold $(M, g)$ the endomorphisms $X \wedge_{A}$ $Y, \mathcal{R}(X, Y)$ and $\mathcal{C}(X, Y)$ by $([7],[11],[17],[18],[19],[21],[22])$

$$
\begin{aligned}
\left(X \wedge_{A} Y\right) Z & =A(Y, Z) X-A(X, Z) Y \\
\mathcal{R}(X, Y) Z & =\left[\nabla_{X}, \nabla_{Y}\right] Z-\nabla_{[X, Y]} Z \\
\mathcal{C}(X, Y) & =\mathcal{R}(X, Y)-\frac{1}{n-2}\left(X \wedge_{g} \mathcal{L} Y+\mathcal{L} X \wedge_{g} Y-\frac{\kappa}{n-1} X \wedge_{g} Y\right)
\end{aligned}
$$

respectively, where $A$ is a $(0,2)$-tensor on $M, X, Y, Z \in \Xi(M), \Xi(M)$ being the Lie algebra of vector fields of $M$. The Ricci operator $\mathcal{L}$ is defined by $S(X, Y)=g(X, \mathcal{L} Y)$, where $S$ is the Ricci tensor and $\kappa$ the scalar curvature of $(M, g)$, respectively. We define the tensor $G$, the Riemannian-Christoffel curvature tensor $R$ and the Weyl conformal curvature tensor $C$ of $(M, g)$ by ([7], $[11],[17],[18],[19],[21],[22])$

$$
\begin{aligned}
& G\left(X_{1}, X_{2}, X_{3}, X_{4}\right)=g\left(\left(X_{1} \wedge_{g} X_{2}\right) X_{3}, X_{4}\right), \\
& R\left(X_{1}, X_{2}, X_{3}, X_{4}\right)=g\left(\mathcal{R}\left(X_{1}, X_{2}\right) X_{3}, X_{4}\right), \\
& C\left(X_{1}, X_{2}, X_{3}, X_{4}\right)=g\left(\mathcal{C}\left(X_{1}, X_{2}\right) X_{3}, X_{4}\right),
\end{aligned}
$$

respectively. For $(0,2)$-tensors $A$ and $B$ we define its Kulkarni-Nomizu product $A \wedge B$ by ([11], [21])

$$
\begin{aligned}
(A \wedge B)\left(X_{1}, X_{2} ; X, Y\right)= & A\left(X_{1}, Y\right) B\left(X_{2}, X\right)+A\left(X_{2}, X\right) B\left(X_{1}, Y\right) \\
& -A\left(X_{1}, X\right) B\left(X_{2}, Y\right)-A\left(X_{2}, Y\right) B\left(X_{1}, X\right) .
\end{aligned}
$$

We note that the Weyl tensor $C$ can be presented in the following form

$$
C=R-\frac{1}{n-2} g \wedge S+\frac{\kappa}{(n-1)(n-2)} G .
$$

For a $(0,2)$-tensor $A$ we define the $(0,4)$-tensor $\bar{A}$ by $\bar{A}=\frac{1}{2} A \wedge A$. Thus we have

$$
\bar{A}\left(X_{1}, X_{2}, X_{3}, X_{4}\right)=A\left(X_{1}, X_{4}\right) A\left(X_{2}, X_{3}\right)-A\left(X_{1}, X_{3}\right) A\left(X_{2}, X_{4}\right) .
$$

For a $(0, k)$-tensor $T, k \geq 1$ and a symmetric $(0,2)$-tensor $A$ we define the $(0, k)$-tensor $A \cdot T$ and the $(0, k+2)$-tensors $R \cdot T$ and $Q(A, T)$ by

$$
\begin{aligned}
& (A \cdot T)\left(X_{1}, \ldots, X_{k}\right) \\
= & -T\left(\mathcal{A} X_{1}, X_{2}, \ldots, X_{k}\right)-\cdots-T\left(X_{1}, X_{2}, \ldots, \mathcal{A} X_{k}\right), \\
& (R \cdot T)\left(X_{1}, \ldots, X_{k} ; X, Y\right) \\
= & (\mathcal{R}(X, Y) \cdot T)\left(X_{1}, \ldots, X_{k}\right) \\
= & -T\left(\mathcal{R}(X, Y) X_{1}, X_{2}, \ldots, X_{k}\right)-\cdots-T\left(X_{1}, \ldots, X_{k-1}, \mathcal{R}(X, Y) X_{k}\right), \\
& Q(A, T)\left(X_{1}, \ldots, X_{k} ; X, Y\right) \\
= & \left(\left(X \wedge_{A} Y\right) \cdot T\right)\left(X_{1}, \ldots, X_{k}\right) \\
= & -T\left(\left(X \wedge_{A} Y\right) X_{1}, X_{2}, \ldots, X_{k}\right)-\cdots-T\left(X_{1}, \ldots, X_{k-1},\left(X \wedge_{A} Y\right) X_{k}\right),
\end{aligned}
$$


where $\mathcal{A}$ is the endomorphism of $\Xi(M)$ defined by $g(\mathcal{A} X, Y)=A(X, Y)$. Putting in the above formulas $T=R, T=S$ or $T=C, A=g$ or $A=S$, we obtain the tensors: $R \cdot R, R \cdot S, R \cdot C, C \cdot S, Q(g, R), Q(g, S), Q(g, C)$, $Q(S, R), Q(S, C), S \cdot R$ and $S \cdot C$.

In a Lorentzian manifold, a vector field $P$ defined by $g(X, P)=A(X)$ for any vector field $X$ is said to be a proper concircular vector field if

$$
\left(\nabla_{X} A\right)(Y)=\alpha\{g(X, Y)+\omega(X) A(Y)\},
$$

where $\alpha$ is a non-zero scalar and $\omega$ is a closed 1-form ([33]).

\section{Some basic properties of $L Q E_{n}$}

In this section some properties of $L Q E_{n}$ are obtained. We prove the following:

Proposition 3.1. In a $L Q E_{n}, n \geq 3$, the following results hold:

(i) $\beta-\alpha$ is the Ricci curvature in the direction of the generator $\rho$.

(ii) The Ricci tensor $S$ has only two distinct eigenvalues $(\alpha-\beta)$ and $\alpha$ of multiplicity 1 and $(n-1)$ respectively.

(iii) If the generator $\rho$ is a parallel vector field, then the associated scalars $\alpha$ and $\beta$ are equal and $Q \rho$ is orthogonal to $\rho$.

(iv) If $\alpha \neq \beta$, then $\rho$ is generic.

Proof. (i) and (ii) easily follow from (1.1).

(iii) If $\rho$ is a parallel vector field, then $\nabla_{X} \rho=0$, where $\nabla$ denotes the operator of covariant differentiation with respect to the Lorentzian metric $g$. And hence $R(X, Y) \rho=0$, which implies that $S(X, \rho)=0$ for all $X$. Consequently, (iii) follows from (1.1).

(iv) It is known that $([31]$, p. 36$)$ if $W$ is a non-null vector on a Lorentzian manifold $M$ with $S(W, W) \neq 0$, then $W$ is generic. Since $\alpha \neq \beta$, it follows from (1.1) that $S(\rho, \rho) \neq 0$ and hence (iv) is proved.

Proposition 3.2 ([31], p. 35). If $W$ is a timelike vector in a Lorentzian manifold $\left(M^{n}, g\right), n \geq 2$, then the following conditions are equivalent:

1. The timelike vector $W$ is generic.

2. At least one plane containing $W$ has non-zero sectional curvature.

3. $R(\cdot, W) W$ is not the trivial map.

In view of Proposition 3.1(iv) and Proposition 3.2, we can state the following:

Theorem 3.1. If a Lorentzian manifold $\left(M^{n}, g\right), n \geq 3$, is a $L(Q E)_{n}$ with distinct associated scalars, then the following conditions are equivalent:

1. At least one plane containing $\rho$ has non-zero sectional curvature.

2. $R(\cdot, \rho) \rho$ is not the trivial map.

Proposition 3.3 ([3], [31], [41]). For a connected orientable manifold $M^{n}$, the following assertions are equivalent:

1. There is a nowhere vanishing vector field $V$ on $M^{n}$. 
2. Either $M^{n}$ is non-compact, or $M^{n}$ is compact and has Euler number $\chi\left(M^{n}\right)=0$.

Proposition 3.4 ([21], Lemma 3.1). Let B be a symmetric (0,2)-tensor on a semi-Riemannian manifold $(M, g), n \geq 3$, and let $\mathcal{U}_{B}$ be the set of all points of $M$ at which $B$ is not proportional to $g$. If at $x \in \mathcal{U}_{B}$ we have

$$
B \wedge B=2 \alpha g \wedge B+2 \beta G, \quad \alpha, \beta \in \mathbb{R},
$$

then $\alpha^{2}=-\beta$ and $\operatorname{rank}(B-\alpha g)=1$ at $x$.

Theorem 3.2. Let $\left(M^{n}, g\right), n \geq 3$, be a connected orientable Lorentzian manifold which is either non-compact or compact with vanishing Euler number. If there is a unit timelike generic vector field $\rho$ on $M$ such that the relation $S=2 \alpha g \wedge S+2 \beta G$ holds with $\alpha^{2}+\beta \neq 0$, then the manifold is a $L Q E_{n}$.

Proof. By virtue of Proposition 3.3, it follows that there is nowhere vanishing vector field $\rho$ on the connected orientable Lorentzian manifold $M$ such that $g(\rho, \rho)=-1$. Since $\rho$ is generic, $S(\rho, \rho) \neq 0$. Hence by virtue of Proposition 3.4, it follows that $\operatorname{rank}(S-\alpha g)=1$ at each point of $M$ in which $S$ is not proportional to $g$. This proves the result.

Theorem 3.3. In a Ricci symmetric $L Q E_{n}, n \geq 3$, the associated scalars are constants and the associated 1-form is closed.

Proof. If a $L Q E_{n}$ is Ricci symmetric, then it follows from (1.1) that

$$
(X \alpha) g(Y, Z)+(X \beta) A(Y) A(Z)+\beta\left[\left(\nabla_{X} A\right)(Y) A(Z)+A(Y)\left(\nabla_{X} A\right)(Z)\right]=0 .
$$

Plugging $\rho$ in place of $Y$ and $Z$ in (3.1), we obtain

$$
(X \alpha)=(X \beta) \text {. }
$$

Again taking an orthonormal frame field at any point of the manifold and then contracting (3.1) over $Y$ and $Z$, we get

$$
n(X \alpha)-(X \beta)=0,
$$

which yields by virtue of (3.2) that

$$
(X \alpha)=(X \beta)=0 \text { for all } X
$$

and hence (3.1) implies that

$$
\left(\nabla_{X} A\right)(Y) A(Z)+A(Y)\left(\nabla_{X} A\right)(Z)=0 .
$$

Setting $Z=\rho$ in the last relation, we have

$$
\left(\nabla_{X} A\right)(Y)=0 \text { for all } X, Y,
$$

which implies that the 1 -form $A$ is closed. This proves the theorem.

Theorem 3.4. If the Ricci tensor of a $L Q E_{n}, n \geq 3$, with distinct associated scalars is of Codazzi type and the associated 1-form $A$ is closed, then the generator of the manifold and the vector field $(\operatorname{grad} \alpha-\operatorname{grad} \beta)$ are co-directional. 
Proof. From (1.1), it follows that

$$
\begin{aligned}
\left(\nabla_{Y} S\right)(X, Z)= & (Y \alpha) g(X, Z)+(Y \beta) A(X) A(Z) \\
& +\beta\left[\left(\nabla_{Y} A\right)(X) A(Z)+A(X)\left(\nabla_{Y} A\right)(Z)\right] .
\end{aligned}
$$

In view of $(3.3)$ we have

$$
\begin{aligned}
\left(\nabla_{X} S\right)(Y, Z)-\left(\nabla_{Y} S\right)(X, Z)= & (X \alpha) g(Y, Z)-(Y \alpha) g(X, Z) \\
& +(X \beta) A(Y) A(Z)-(Y \beta) A(X) A(Z) \\
& +\beta\left[\left\{\left(\nabla_{X} A\right)(Y)-\left(\nabla_{Y} A\right)(X)\right\} A(Z)\right. \\
& \left.+A(Y)\left(\nabla_{X} A\right)(Z)-A(X)\left(\nabla_{Y} A\right)(Z)\right] .
\end{aligned}
$$

If the Ricci tensor is of Codazzi type [20], that is, if

$$
\left(\nabla_{X} S\right)(Y, Z)=\left(\nabla_{Y} S\right)(X, Z) \text { for all } X, Y, Z,
$$

then (3.4) yields

$$
\begin{aligned}
& (X \alpha) g(Y, Z)-(Y \alpha) g(X, Z)+(X \beta) A(Y) A(Z) \\
& -(Y \beta) A(X) A(Z)+\beta\left[\left\{\left(\nabla_{X} A\right)(Y)-\left(\nabla_{Y} A\right)(X)\right\} A(Z)\right. \\
& \left.+A(Y)\left(\nabla_{X} A\right)(Z)-A(X)\left(\nabla_{Y} A\right)(Z)\right]=0 .
\end{aligned}
$$

We suppose that the 1 -form $A$ is closed. Then $d A(X, Y)=0$ and hence

$$
\left(\nabla_{X} A\right)(Y)=\left(\nabla_{Y} A\right)(X) \text { for all } X, Y \text {. }
$$

Using (3.7), it follows from (3.6) that

$$
\begin{aligned}
& (X \alpha) g(Y, Z)-(Y \alpha) g(X, Z)+(X \beta) A(Y) A(Z) \\
& -(Y \beta) A(X) A(Z)+\beta\left[A(Y)\left(\nabla_{X} A\right)(Z)-A(X)\left(\nabla_{Y} A\right)(Z)\right]=0 .
\end{aligned}
$$

Putting $Z=\rho$ in (3.8) we get

$$
D(X) A(Y)=A(X) D(Y),
$$

which implies that $\rho$ and $\mu$ are co-directional, where

$$
D(X)=g(X, \mu)=g(X, \operatorname{grad} \alpha-\operatorname{grad} \beta) \text { for all } X .
$$

This proves the theorem.

\section{Conformally flat $L Q E_{n}$}

As a generalization of the manifold of constant curvature, the notion of the manifold of quasi-constant curvature arose during the study of conformally flat hypersurfaces by Chen and Yano [4].

Definition 4.1. A Lorentzian manifold $\left(M^{n}, g\right), n>3$, is said to be of quasiconstant curvature [4] if it is conformally flat and its curvature tensor $R$ of type $(0,4)$ is of the form:

$$
R=a G+b g \wedge B
$$

where $a, b$ are scalars of which $b \neq 0$, and $B=A \otimes A, A$ being a non-zero 1-form. Such an $n$-dimensional manifold is denoted by the symbol $L Q C_{n}$. 
Theorem 4.1. Every conformally flat $L Q E_{n}, n>3$, is a $L Q C_{n}$.

Proof. Since the manifold under consideration is conformally flat, (2.1) implies that

$$
R=\frac{1}{n-2} g \wedge S-\frac{\kappa}{(n-1)(n-2)} G
$$

which yields by virtue of (1.1) that (4.1) holds, where $a=\frac{(n-2) \alpha+\beta}{(n-1)(n-2)}$ and $b=\frac{\beta}{n-2}$. This proves the theorem.

Corollary 4.1. Every $L Q E_{3}$ is a $L Q C_{3}$.

From (4.1), it follows that

$$
S=\gamma g+\delta A \otimes A,
$$

where $\gamma=(n-1) a-b$ and $\delta=(n-2) b$. This leads to the following:

Theorem 4.2. Every $L Q C_{n}, n>3$, is a $L Q E_{n}$.

Lemma 4.1. In a conformally flat $L Q E_{n}, n>3$, the curvature tensor $R$ has the following properties:

(i) $R=a G$,

(ii) $R(X, \rho) Y=-a g(X, Y) \rho$ and

(iii) $R(X, \rho) \rho=-a X$

for all vector fields $X, Y \in \rho^{\perp}$, the $(n-1)$-dimensional distribution orthogonal to the generator $\rho$, where $a=\frac{(n-2) \alpha+\beta}{(n-1)(n-2)}$.

Proof. If $\rho^{\perp}$ is the $(n-1)$-dimensional distribution orthogonal to the generator $\rho$, then $g(X, \rho)=0$ if and only if $X \in \rho^{\perp}$. Hence the lemma follows from (4.2).

Theorem 4.3. In a conformally flat $L Q E_{n}, n>3$, the sectional curvature of all planes determined by the vectors $X, Y \in \rho^{\perp}$ and the sectional curvature of all planes determined by the vectors $X$ and $\rho$, where $X \in \rho^{\perp}$ are equal and each of them is a.

Proof. If $K_{1}$ is the sectional curvature of the plane determined by $X, Y \in \rho^{\perp}$, then by virtue of Lemma 4.1(i) we obtain $K_{1}=a$.

Also if $K_{2}$ is the sectional curvature of the plane determined by $X$ and $\rho$, where $X \in \rho^{\perp}$, then by virtue of Lemma 4.1(iii), we obtain $K_{2}=a$. This proves the theorem.

We note that $K_{1}$ and $K_{2}$ are constants if and only if $(n-2) \alpha+\beta$ is constant. Hence we can state the following:

Corollary 4.2. In a conformally flat $L Q E_{n}, n>3$, the sectional curvature $K_{1}$ of all planes determined by $X$ and $Y$ as well as the sectional curvature $K_{2}$ of all planes determined by $X$ and $\rho$ are constants if and only if $(n-2) \alpha+\beta$ = constant. 


\section{General relativistic viscous fluid $L Q E_{4}$ spacetime}

A viscous fluid spacetime is a connected Lorentzian manifold $\left(M^{4}, g\right)$ with signature $(-,+,+,+)$. General relativity flows from Einstein's equation given by

$$
S+\left(\lambda-\frac{\kappa}{2}\right) g=K T,
$$

where $\kappa$ is the scalar curvature, $k$ is the gravitational constant, $\lambda$ is the cosmological constant and $T$ is the energy-momentum tensor of type $(0,2)$.

We now consider a perfect fluid spacetime. Then the energy-momentum tensor is of the form ([31]):

$$
T=p g+(\sigma+p) A \otimes A,
$$

where $\sigma, p$ are respectively the energy density, isotropic pressure and $\rho$ is the unit timelike flow vector field of the fluid such that $A(\cdot)=g(\cdot, \rho)$.

Then by virtue of (5.2), (5.1) can be written as

$$
S=\alpha g+\beta A \otimes A,
$$

where $\alpha=\left(\frac{\kappa}{2}+K p-\lambda\right)$ and $\beta=K(\sigma+p)$. This shows that the spacetime under consideration is a $L Q E_{4}$ with $\alpha=\frac{\kappa}{2}+K p-\lambda, \beta=K(\sigma+p)$ as associated scalars, $A$ as the associated 1-form with generator $\rho$.

Conversely, we consider a $L Q E_{4}$. Then in view of (1.1), (5.1) takes the form (5.2), where $p=\frac{\alpha+\lambda-\frac{\kappa}{2}}{K}$ and $\sigma=\frac{\beta-\alpha-\lambda+\frac{\kappa}{2}}{K}$. Consequently, a $L Q E_{4}$ is a perfect fluid spacetime. Hence we can state the following:

Theorem 5.1. Let $M$ be a 4-dimensional connected Lorentzian manifold obeying Einstein's equation with a cosmological constant. Then $M$ is a perfect fluid spacetime if and only if it is a $L Q E_{4}$.

A connected $L Q E_{4}$ is said to be a quasi-Einstein spacetime. Let us consider a quasi-Einstein spacetime obeying Einstein's equation with a cosmological constant. Then we have

$$
S=\left(\frac{\kappa}{2}+K p-\lambda\right) g+K(\sigma+p) A \otimes A,
$$

which, in view of (1.1), yields

$$
\sigma=\frac{2 \alpha+\beta-2 \lambda}{2 K}, \quad p=\frac{2 \lambda+\beta-2 \alpha}{2 K} .
$$

From (5.4), it follows that $\sigma$ and $p$ are constants if and only if $\alpha$ and $\beta$ are constants.

Hence we can state the following:

Theorem 5.2. If a viscous fluid quasi-Einstein spacetime with non-constant associated scalars obeys Einstein's equation with a cosmological constant, then none of the energy density and isotropic pressure of the fluid can be a constant. 
We suppose that, in a quasi-Einstein spacetime, the isotropic pressure $p$ is positive. Then since $\sigma>0$, it follows from (5.4) that

$$
\alpha-\frac{\beta}{2}<\lambda<\alpha+\frac{\beta}{2} \text {. }
$$

This leads to the following:

Theorem 5.3. If a viscous fluid quasi-Einstein spacetime with positive isotropic pressure obeys Einstein's equation with a cosmological constant $\lambda$, then $\lambda$ satisfies the relation (5.5).

We now discuss whether a viscous fluid quasi-Einstein spacetime with generator $\rho$ as the unit timelike flow vector field can admit heat flux or not. Therefore, if possible, let the energy-momentum tensor $T$ be of the following form [30]:

$$
T=p g+(\sigma+p) A \otimes A+A \otimes B+B \otimes A+D,
$$

where $B(\cdot)=g(\cdot, \mu) ; \mu$ being the heat flux vector field. Then we have $g(\rho, \mu)=$ 0 , i.e., $B(\rho)=0$. Hence by virtue of $(5.6)$, (5.1) reduces to

$$
S+\left(\lambda-\frac{\kappa}{2}-K p\right) g=K[(\sigma+p) A \otimes A+A \otimes B+B \otimes A],
$$

which yields by virtue of (1.1) that

$$
\left(\alpha-\beta-\frac{\kappa}{2}+K \sigma+\lambda\right) A=-K B .
$$

Since $B(\rho)=0$, the above relation implies that

$$
\alpha-\beta-\frac{\kappa}{2}+K \sigma+\lambda=0
$$

and hence $B=0$. Thus we have the following:

Theorem 5.4. A viscous fluid quasi-Einstein spacetime can not admit heat flux.

We now prove the following:

Theorem 5.5. If the energy-momentum tensor of a viscous fluid quasi-Einstein spacetime is covariantly constant, then the spacetime is Ricci symmetric and its associated scalars $\alpha$ and $\beta$ are constants.

Proof. By the assumption of the theorem, we have $\nabla T=0$, and hence (5.1) yields

$$
\left(\nabla_{Z} S\right)(X, Y)-\frac{d \kappa(Z)}{2} g(X, Y)=0 .
$$

Taking contraction over $X$ and $Y$ we get

$$
d \kappa(Z)=0 \text { for all } Z,
$$


which shows that the scalar curvature is constant.

From (5.7) and (5.8), it follows that $\nabla S=0$ and hence the spacetime is Ricci symmetric. Consequently, from (1.1) it follows that

$d \alpha(Z) g(X, Y)+d \beta(Z) A(X) A(Y)+\beta\left[\left(\nabla_{Z} A\right)(X) A(Y)+A(X)\left(\nabla_{Z} A\right)(Y)\right]=0$.

Taking contraction over $X$ and $Y$ we get

$$
4 d \alpha(Z)-d \beta(Z)=0 .
$$

Again setting $X=Y=\rho$ in (5.9) we obtain

$$
d \alpha(Z)=d \beta(Z)
$$

From (5.10) and (5.11) it follows that $\alpha$ and $\beta$ are constants. This proves the theorem.

Theorem 5.6. If the energy-momentum tensor of a viscous fluid quasi-Einstein spacetime is of Codazzi type, then the following properties hold:

(i) The Ricci tensor of the spacetime is of Codazzi type and it is of constant scalar curvature.

(ii) The integral curves of the flow vector field are geodesics.

(iii) The flow vector field of the fluid is irrotational.

(iv) The acceleration vector of the fluid must vanish but the expansion scalar will never vanish.

(v) The associated 1-form of the spacetime is closed and the flow vector field of the fluid is a unit proper concircular vector field.

(vi) If the flow vector field satisfies $\nabla_{X} \rho=f \phi X$ for all $X, f$ being a smooth function on $M$ and $\phi$ being an $(1,1)$ tensor field, then the spacetime is a concircular structure spacetime.

Proof. (i) If the energy-momentum tensor of a viscous fluid quasi-Einstein spacetime is of Codazzi type tensor [20], then we have

$$
\left(\nabla_{X} T\right)(Y, Z)=\left(\nabla_{Z} T\right)(Y, X) .
$$

From (5.1) we get

$$
\left(\nabla_{Z} S\right)(X, Y)-\frac{1}{2} d \kappa(Z) g(X, Y)=K\left(\nabla_{Z} T\right)(X, Y)
$$

which yields by virtue of (5.12) that

$$
\left(\nabla_{X} S\right)(Y, Z)-\left(\nabla_{Z} S\right)(X, Y)-\frac{1}{2}[d \kappa(X) g(Y, Z)-d \kappa(Z) g(X, Y)]=0 .
$$

Contracting over $Y$ and $Z$ we get $d \kappa(X)=0$ for all $X$ and hence (5.14) takes the form

$$
\left(\nabla_{X} S\right)(Y, Z)=\left(\nabla_{Z} S\right)(X, Y)
$$

Hence the Ricci tensor is of Codazzi type. 
(ii) In view of (1.1), (5.15) takes the form

$$
\begin{gathered}
d \alpha(X) g(Y, Z)+d \beta(X) A(Y) A(Z) \\
+\beta\left[\left(\nabla_{X} A\right)(Y) A(Z)+A(Y)\left(\nabla_{X} A\right)(Z)\right] \\
=\quad d \alpha(Z) g(X, Y)+d \beta(Z) A(X) A(Y) \\
\quad+\beta\left[\left(\nabla_{Z} A\right)(X) A(Y)+A(X)\left(\nabla_{Z} A\right)(Y)\right] .
\end{gathered}
$$

Let $\left\{e_{i} \mid i=1,2,3,4\right\}$ be an orthonormal frame field at any point of the quasiEinstein spacetime. Setting $Y=Z=e_{i}$ in (5.16) and then taking summation for $1 \leq i \leq 4$, we obtain

$$
3 d \alpha(X)-d \beta(X)=d \beta(\rho) A(X)+\beta\left\{\left(\nabla_{\rho} A\right)(X)+A(X) \sum_{i=1}^{4} \varepsilon_{i}\left(\nabla_{e_{i}} A\right)\left(e_{i}\right)\right\},
$$

where $\varepsilon_{i}=g\left(e_{i}, e_{i}\right)$. Again setting $Y=Z=\rho$ in (5.16) we get

$$
d \beta(X)-d \alpha(X)=d \alpha(\rho) A(X)-d \beta(\rho) A(X)-\beta\left(\nabla_{\rho} A\right)(X) .
$$

Adding (5.17) and (5.18) we have

$$
2 d \alpha(X)=d \alpha(\rho) A(X)+\beta A(X) \sum_{i=1}^{4} \varepsilon_{i}\left(\nabla_{e_{i}} A\right)\left(e_{i}\right) .
$$

Setting $X=\rho$ in (5.19) we get

$$
-3 d \alpha(\rho)=\beta \sum_{i=1}^{4} \varepsilon_{i}\left(\nabla_{e_{i}} A\right)\left(e_{i}\right) .
$$

By virtue of (5.23) and (5.24) we obtain

$$
d \alpha(X)+d \alpha(\rho) A(X)=0 .
$$

Since in a quasi-Einstein spacetime the scalar curvature $\kappa$ is given by $\kappa=4 \alpha-\beta$, so by virtue of (5.19) we get $d \alpha(X)=\frac{1}{4} d \beta(X)$, and hence (5.21) yields

$$
d \beta(X)+d \beta(\rho) A(X)=0 .
$$

In view of $(5.20),(5.21)$ and $(5.22)$, we obtain from (5.17) that

$$
\left(\nabla_{\rho} A\right)(X)=0
$$

for all $X$, which implies that $\nabla_{\rho} \rho=0$ and hence the integral curves of $\rho$ are geodesics.

(iii) Substituting $Z$ by $\rho$ in (5.16) and then using (5.22) and (5.23) we obtain

$$
\beta\left(\nabla_{X} A\right)(Y)=d \alpha(X) A(Y)-d \alpha(\rho) g(X, Y) .
$$

From (5.21) and (5.24), it follows that

$$
\left(\nabla_{X} A\right)(Y)=f\{g(X, Y)+A(X) A(Y)\},
$$


where $f$ is a non-vanishing scalar given by

$$
f=-\frac{d \alpha(\rho)}{\beta}=-\frac{d \beta(\rho)}{4 \beta} .
$$

The relation (5.25) implies that 1-form $A$ is closed. Again from (5.25), it follows that

$$
g\left(\nabla_{X} \rho, Y\right)-g\left(X, \nabla_{Y} \rho\right)=0 .
$$

This means that the flow vector field $\rho$ is irrotational.

(iv) We have $\operatorname{div} \rho=\sum_{i=1}^{4} \varepsilon_{i} g\left(\nabla_{e_{i}} \rho, e_{i}\right)$, where $\varepsilon_{i}=g\left(e_{i}, e_{i}\right),\left\{e_{i}\right\}, i=$ $1,2,3,4$ is an orthonormal frame field, 'div' denotes the divergence. From (5.25) we have

$$
g\left(\nabla_{X} \rho, Y\right)=f\{g(X, Y)+A(X) A(Y)\},
$$

which yields $\operatorname{div} \rho=3 f \neq 0$ as $f \neq 0$. Since $\operatorname{div} \rho$ represents the expansion scalar and $\nabla_{\rho} \rho$ represents the acceleration vector, (iv) is proved.

(v) From (5.26) we have

$$
\nabla_{X} f=\frac{1}{4 \beta^{2}} d \beta(\rho) d \beta(X)-\frac{1}{4 \beta} d^{2} \beta(\rho, X) .
$$

From (5.22), it follows that

$$
d^{2} \beta(X, Y)=-d^{2} \beta(\rho, Y) A(X)-d \beta(\rho)\left(\nabla_{Y} A\right)(X) .
$$

Since in a Lorentzian manifold $M$ for any scalar function $h \in C^{\infty}(M)$, the relation $d^{2} h(X, Y)=d^{2} h(Y, X)$ holds for all $X, Y$, from (5.28) it follows by virtue of $(5.25)$ that

$$
d^{2} \beta(\rho, X) A(Y)-d^{2} \beta(\rho, Y) A(X)=0,
$$

which yields

$$
d^{2} \beta(\rho, X)=\psi A(X),
$$

where $\psi=-d^{2} \beta(\rho, \rho)$ is a non-vanishing scalar. Hence, (5.27) reduces to

$$
\nabla_{X} f=\lambda A(X)
$$

where $\lambda=-\left\{\left(\frac{d \beta(\rho)}{2 \beta}\right)^{2}+\frac{\psi}{4 \beta}\right\}$ is a non-zero scalar. From (5.25), (5.26) and (5.29), it follows that the vector field $\rho$ is a unit proper concircular vector field.

(vi) A 4-dimensional connected Lorentzian manifold admitting a unit timelike proper concircular vector field $\xi$ and satisfying $\nabla_{X} \xi=\gamma \phi X, \phi$ being an $(1,1)$ tensor field and $\gamma$ a non-zero scalar, is a concircular structure spacetime introduced and studied by Shaikh and Baishya ([38], [39]). Hence a quasiEinstein spacetime with Codazzi type energy momentum tensor is a concircular structure spacetime. 


\section{Some examples of $L Q E_{n}$}

This section deals with several non-trivial examples of $L Q E_{n}$.

Example 6.1. In 1989 K. Matsumoto [26] introduced the notion of LPSasakian manifolds. Then I. Mihai and R. Rosca [28] introduced the same notion independently and later studied by many authors ([27], [29], [40]).

An $n$-dimensional smooth manifold $M$ is said to be an LP-Sasakian manifold ([6], [28]) if it admits an $(1,1)$ tensor field $\phi$, a vector field $\xi$, an 1-form $\eta$ and a Lorentzian metric $g$, which satisfy

$$
\begin{gathered}
\eta(\xi)=-1, g(X, \xi)=\eta(X), \phi^{2}=I+\eta \otimes \xi, \nabla_{X} \xi=\phi X, \\
\left(\nabla_{X} \phi\right)(Y)=g(X, Y) \xi+\eta(Y) X+2 \eta(X) \eta(Y) \xi,
\end{gathered}
$$

where $\nabla$ denotes the operator of covariant differentiation with respect to the Lorentzian metric $g$.

In an $n$-dimensional LP-Sasakian manifold $M^{n}(\phi, \xi, \eta, g)$, if we put

$$
\Omega(X, Y)=g(\phi X, Y),
$$

then the tensor field $\Omega$ is a symmetric $(0,2)$ tensor field, satisfying the relation $\left(\nabla_{X} \eta\right)(Y)=\Omega(X, Y)$ and $\Omega(X, \xi)=0$ for all $X, Y$.

(i) An LP-Sasakian manifold $\left(M^{n}, g\right)$ is said to be locally $\phi$-symmetric in the strong sense or strongly locally $\phi$-symmetric [36] if it satisfies

$$
\phi^{2}\left(\left(\nabla_{W} R\right)(X, Y) Z\right)=0
$$

for any vector fields $X, Y, Z, W$ tangent to $M$.

Especially, if the vector fields $X, Y, Z, W$ are orthogonal to $\xi$, then it is said to be weakly locally $\phi$-symmetric [36], which is the notion of local $\phi$-symmetry in the sense of Takahashi [42].

In [36] (Theorem 4.4) it is shown that a strongly locally $\phi$-symmetric LPSasakian manifold $\left(M^{n}, g\right)(n>3)$ with $\xi$ as the harmonic vector field is an $\eta$-Einstein manifold, and its Ricci tensor is of the form

$$
S=-(n-1) g-2(n-1) \eta \otimes \eta .
$$

It can be easily shown that such an LP-Sasakian manifold is non-conformally flat. Hence a strongly locally $\phi$-symmetric LP-Sasakian manifold with the timelike vector field $\xi$ as the harmonic vector field is a $L Q E_{n}$ which is not conformally flat.

(ii) Again, generalizing the notion of strong local $\phi$-symmetry, recently, Shaikh et al. [35] introduced and studied the notion of strongly locally $\phi$ recurrent LP-Sasakian manifold.

An LP-Sasakian manifold $\left(M^{n}, g\right)$ is said to be strongly locally $\phi$-recurrent [35] if and only if there exists a non-zero 1-form $A$ such that

$$
\phi^{2}\left(\left(\nabla_{W} R\right)(X, Y) Z\right)=A(W) R(X, Y) Z
$$

for any vector fields $X, Y, Z, W$ tangent to $M$. 
Especially, if $X, Y, Z, W$ are horizontal vector fields, then it is called weakly locally $\phi$-recurrent LP-Sasakian manifold [35].

It is shown that [35] (Theorem 4.3) in a strongly locally $\phi$-recurrent LPSasakian manifold, the timelike vector field $\xi$ is harmonic and hence such a manifold is always an $\eta$-Einstein manifold [35] (Theorem 4.5), which can be easily shown that not conformally flat. Hence a strongly locally $\phi$-recurrent LP-Sasakian manifold is a non-conformally flat $L Q E_{n}$.

(iii) Again Shaikh and Baishya [37] studied Ricci generalized pseudo symmetric [9] LP-Sasakian manifolds and proved that [37] (Theorem 3.1) such a non-Einstein LP-Sasakian manifold is an $\eta$-Einstein manifold which is not conformally flat. Therefore a non-Einstein Ricci generalized pseudosymmetric LP-Sasakian manifold is a non-conformally flat $L Q E_{n}$.

Example 6.2. In 1996, T. Ikawa and M. Erdogan [24] studied the Sasakian manifold with Lorentzian metric. For example, an odd dimensional de Sitter space and Göedel universe have Sasakian structure with Lorentzian metric [24].

A $(2 n+1)$-dimensional differentiable manifold $M^{2 n+1}(n \geq 1)$ of class $C^{\infty}$ equipped with an $(1,1)$ tensor field $\phi$, a global vector field $\xi$ and an 1 -form $\eta$, which satisfy

$$
\phi^{2} X=-X+\eta(X) \xi, \quad \phi \xi=0, \quad \eta(\phi X)=0, \quad \eta(\xi)=1
$$

for any vector field $X$, is called an almost contact manifold with an almost contact structure $(\phi, \xi, \eta)$. The almost contact structure is said to be normal if

$$
N+d \eta \otimes \xi=0,
$$

where $N$ denotes the Nijenhuis tensor formed with $\phi$ [24]. Since $M^{2 n+1}$ has a globally defined vector field $\xi$ without zeros, it is able to have a Lorentzian metric $g$ such that $g(\xi, \xi)=-1$. In fact, if $h$ is an arbitrary Riemannian metric on $M^{2 n+1}$, then $g=h-2 \eta \otimes \eta$ is a desired Lorentzian metric. If $M^{2 n+1}$ has the normal almost contact structure $(\phi, \xi, \eta)$ and the Lorentzian metric $g$ with

$$
g(\phi X, \phi Y)=g(X, Y)+\eta(X) \eta(Y),\left(\nabla_{X} \eta\right)(Y)=g(\phi X, Y),
$$

where $\nabla$ is the covariant derivative with respect to $g$, then $M^{2 n+1}(\phi, \xi, \eta, g)$ is called a Sasakian manifold with the Lorentzian metric [24]. In [24] Ikawa and Erdogan also defined the notion of a $D$-connection on $M^{2 n+1}(\phi, \xi, \eta, g)$ as a linear connection given by

$$
D_{X} Y=\nabla_{X} Y+A(X) Y,
$$

where $\nabla$ is the Lorentzian connection and $A$ is a tensor field of type $(1,2)$ defined by

$$
A(X) Y=g(\phi X, Y) \xi+k \eta(X) \phi Y+\eta(Y) \phi X,
$$

$k$ being an arbitrary fixed non-zero real number. Then, the authors of [24] obtained the various properties of such a $D$-connection. Also, it is shown 
that [24] the Ricci tensor $S^{D}$ of $D$-connection and the Ricci tensor $S$ of the Lorentzian connection satisfies

$$
S^{D}(X, Y)=S(X, Y)-2 k g(X, Y)-2(n+k) \eta(X) \eta(Y) .
$$

Hence, if the Ricci tensor $S^{D}$ of the $D$-connection vanishes, then the Ricci tensor $S$ of the Lorentzian connection takes the form

$$
S=\alpha g+\beta \eta \otimes \eta,
$$

where $\alpha=2 k$ and $\beta=2(n+k)$ are non-vanishing constants. Hence, if the Ricci tensor of the $D$-connection on a Sasakian manifold with a Lorentzian metric vanishes, then it is a $L Q E_{n}$, which is non-conformally flat.

Example 6.3 ([2], [23]). A 3-dimensional Lorentzian contact metric manifold with $Q \phi=\phi Q$ is a $L Q E_{3}$ which is non-conformally flat.

Example 6.4. As a generalization of LP-Sasakian manifold, recently Shaikh [34] introduced the notion of Lorentzian concircular structure manifold and proved its existence and also obtained several applications to the general relativity and cosmology.

Let $M^{n}$ be a Lorentzian manifold admitting a unit timelike concircular vector field $\xi$, called the characteristic vector field of the manifold. Then we have $g(\xi, \xi)=-1$. Since $\xi$ is a unit concircular vector field, there exists a non-zero 1-form $\eta$ such that for $g(X, \xi)=\eta(X)$, the equation of the following form holds

$$
\left(\nabla_{X} \eta\right)(Y)=\alpha\{g(X, Y)+\eta(X) \eta(Y)\}(\alpha \neq 0)
$$

for all vector fields $X, Y$ where $\nabla$ denotes the operator of covariant differentiation with respect to the Lorentzian metric $g$, and $\alpha$ is a non-zero scalar function satisfies

$$
\nabla_{X} \alpha=(X \alpha)=\rho \eta(X),
$$

$\rho$ being a certain scalar function. If we put

$$
\phi X=\frac{1}{\alpha} \nabla_{X} \xi
$$

then from (6.1) we have

$$
\phi X=X+\eta(X) \xi,
$$

from which it follows that $\phi$ is a symmetric $(1,1)$ tensor. Thus the Lorentzian manifold $M^{n}$ together with the unit timelike concircular vector field $\xi$, its associated 1-form $\eta$ and $(1,1)$ tensor field $\phi$ is said to be a Lorentzian concircular structure manifold (briefly $(L C S)_{n}$-manifold) [34].

Especially, if we take $\alpha=1$, then we can obtain the LP-Sasakian structure of Matsumoto [26].

In a $(L C S)_{n}$ manifold, the following relations hold [34]:

$$
\begin{gathered}
R(X, Y) \xi=\left(\rho-\alpha^{2}\right)\{\eta(Y) X-\eta(X) Y\}, \\
S(X, \xi)=(n-1)\left(\rho-\alpha^{2}\right) \eta(X) \text { for any } X, Y .
\end{gathered}
$$


We now consider a $(L C S)_{n}(n>3)$ satisfying the condition

$$
Q(S, R)=0,
$$

where $Q(S, R)$ is defined by $([9],[23])$

$$
\begin{aligned}
Q(S, R)= & (S(X, Y) \cdot R)(U, V) W=\left(\left(X \wedge_{S} Y\right) \cdot R\right)(U, V) W \\
= & \left(X \wedge_{S} Y\right) R(U, V) W-R\left(\left(X \wedge_{S} Y\right) U, V\right) W \\
& -R\left(U,\left(X \wedge_{S} Y\right) V\right) W-R(U, V)\left(X \wedge_{S} Y\right) W .
\end{aligned}
$$

Here the endomorphism $\left(X \wedge_{S} Y\right)$ is defined by

$$
\left(X \wedge_{S} Y\right) Z=S(Y, Z) X-S(X, Z) Y .
$$

In view of (6.5) and (6.7), it follows from (6.6) that

$$
\begin{aligned}
& (n-1)\left(\rho-\alpha^{2}\right)\{\eta(R(U, V) W) X-\eta(U) R(X, V) W \\
& -\eta(V) R(U, X) W-\eta(W) R(U, V) X\}-S(X, R(U, V) W) \xi \\
& +S(X, U) R(\xi, V) W+S(X, V) R(U, \xi) W+S(X, W) R(U, V) \xi=0 .
\end{aligned}
$$

Taking the inner product on both sides of (6.8) by $\xi$, we get

$$
\begin{aligned}
& (n-1)\left(\rho-\alpha^{2}\right)\{\eta(R(U, V) W) \eta(X)-\eta(U) \eta(R(X, V) W) \\
& -\eta(V) \eta(R(U, X) W)-\eta(W) \eta(R(U, V) X)\}+S(X, R(U, V) W) \\
& +S(X, U) \eta(R(\xi, V) W)+S(X, V) \eta(R(U, \xi) W)=0 .
\end{aligned}
$$

Putting $W=\xi$ in (6.9), we obtain

$$
S(X, R(U, V) \xi)=(n-1)\left(\rho-\alpha^{2}\right) \eta(R(U, V) X) .
$$

Again replacing $U=\xi$ in (6.10) and using (6.3) and (6.4), we get

$$
S(X, V)=-(n-1)\left(\rho-\alpha^{2}\right) g(X, V)-2(n-1)\left(\rho-\alpha^{2}\right) \eta(X) \eta(V) .
$$

It can be easily shown that such an $(L C S)_{n}$ manifold is not conformally flat. Hence a $(L C S)_{n}(n>3)$ manifold with $Q(S, R)=0$ is a $L Q E_{n}$ which is nonconformally flat.

Example 6.5. We define a Lorentzian metric $g$ on a 5 -dimensional real number space $\mathbb{R}^{5}=\left\{\left(x^{\alpha}, y^{\alpha}, z\right): \alpha=1,2\right\}$ by the formula

$$
\begin{aligned}
d s^{2}=g_{i j} d x^{i} d x^{j}= & \frac{1}{4}\left\{1+\left(y^{1}\right)^{2}\right\}\left(d x^{1}\right)^{2}+\frac{1}{2} d x^{1} d x^{2} \\
& -\frac{1}{2} y^{1} d x^{1} d z+\frac{1}{4}\left\{1+\left(y^{2}\right)^{2}\right\}\left(d x^{2}\right)^{2} \\
& -\frac{1}{2} y^{2} d x^{2} d z-\frac{1}{4}\left(d y^{1}\right)^{2} \\
& +\frac{1}{4}\left(d y^{2}\right)^{2}+\frac{1}{4}(d z)^{2},
\end{aligned}
$$


where $i, j=1,2,3,4,5$ and $x^{\alpha^{*}} \equiv x^{2+\alpha}=y^{\alpha}, x^{\sigma}=z, \sigma=5$. Then the only non-vanishing components of the Christoffel symbols and the curvature tensor are

$$
\begin{aligned}
& \Gamma_{\alpha \beta^{*}}^{\mu}=\frac{1}{2} \delta_{\mu \beta} y^{\alpha}, \Gamma_{\alpha \beta}^{\mu^{*}}=-\frac{1}{2}\left(\delta_{\alpha \mu} y^{\beta}+\delta_{\mu \beta} y^{\alpha}\right), \Gamma_{\alpha \sigma}^{\mu^{*}}=\frac{1}{2} \delta_{\alpha \mu}, \\
& \Gamma_{\alpha \beta^{*}}^{\sigma}=\frac{1}{2}\left(y^{\alpha} y^{\beta}-\delta_{\alpha \beta}\right), \Gamma_{\sigma \beta^{*}}^{\sigma}=-\frac{1}{2} y^{\beta}, \Gamma_{\alpha^{*} \sigma}^{\mu}=-\frac{1}{2} \delta_{\alpha \mu}, \\
& R_{\mu \gamma \beta \alpha}=\frac{1}{16}\left(\delta_{\alpha \beta} y^{\beta} y^{\gamma}+\delta_{\beta \gamma} y^{\alpha} y^{\mu}-\delta_{\alpha \gamma} y^{\beta} y^{\mu}-\delta_{\beta \mu} y^{\alpha} y^{\gamma}\right), \\
& R_{\mu^{*} \gamma^{*} \beta \alpha}=\frac{1}{16}\left(\delta_{\alpha \gamma} \delta_{\beta \mu}-\delta_{\alpha \mu} \delta_{\beta \gamma}\right), R_{\mu \gamma \sigma \alpha}=\delta_{\alpha \gamma} y^{\sigma}-\delta_{\alpha \sigma} y^{\gamma}, \\
& R_{\mu \gamma^{*} \beta^{*} \alpha}=\frac{1}{16}\left(\delta_{\beta \gamma} y^{\alpha} y^{\mu}-2 \delta_{\alpha \beta} \delta_{\gamma \sigma}-\delta_{\alpha \gamma} \delta_{\beta \sigma}\right), \\
& R_{\mu \sigma \sigma \alpha}=\frac{1}{16} \delta_{\alpha \mu}, R_{\sigma \gamma^{*} \sigma \alpha^{*}}=-\frac{1}{16} \delta_{\alpha \gamma}, R_{\mu^{*} \sigma \beta^{*} \alpha}=\frac{1}{16} \delta_{\beta \mu} y^{\alpha},
\end{aligned}
$$

and the components which can be obtained from these by the symmetry properties. Using the above relations, we can find the non-vanishing components of Ricci tensor as follows:

$$
\left\{\begin{array}{l}
S_{\mu \alpha}=-\frac{1}{2}\left(\delta_{\alpha \mu}-2 y^{\alpha} y^{\mu}\right), S_{\mu^{*} \alpha^{*}}=\frac{1}{2} \delta_{\alpha \mu}, \\
S_{\sigma \sigma}=1, S_{\gamma \sigma}=-y^{\gamma}
\end{array}\right.
$$

Also it can be easily found that the scalar curvature of the manifold is non-zero. Therefore $\mathbb{R}^{5}$ with the considered metric is a Lorentzian manifold $\left(M^{5}, g\right)$ of non-vanishing scalar curvature. We shall now show that this $M^{5}$ is a $L Q E_{5}$, i.e., it satisfies (1.1).

If we consider

$$
A=\sqrt{\frac{3}{2 b}}\left(\sum_{\alpha=1}^{2} y^{\alpha} d x^{\alpha}-d z\right),
$$

where $b$ is a scalar, then we have

$$
A_{i}=\left(\sqrt{\frac{3}{2 b}} y^{\alpha}, 0,0,-\sqrt{\frac{3}{2 b}}\right) .
$$

From (6.11)-(6.13), it follows that

$$
S_{i j}=2 g_{i j}-b A_{i} A_{j} .
$$

Therefore, $\left(\mathbb{R}^{5}, g\right)$ is a $L Q E_{5}$. Hence we can state the following:

Theorem 6.1. Let $\left(M^{5}, g\right)$ be a Lorentzian manifold endowed with the metric given in (6.11). Then $\left(M^{5}, g\right)$ is a non-conformally flat $L Q E_{5}$.

Acknowledgement. The authors are grateful to the referee for the valuable suggestions and comments towards the improvement of the paper. 


\section{References}

[1] P. Amendt and H. Weitzner, Relativistically covariant warm charged fluid beam modeling, The Physics of Fluids 28 (1985), 949-957.

2] D. E. Blair, T. Koufogiorgos, and R. Sharma, A classification of 3-dimensional contact metric manifolds with $Q \phi=\phi Q$, Kodai Math. J. 13 (1990), no. 3, 391-401.

[3] J. K. Beem and P. E. Ehrlich, Global Lorentzian Geometry, Marcel Dekker, 1981.

[4] B. Y. Chen and K. Yano, Hypersurfaces of a conformally flat space, Tensor (N.S.) 26 (1972), 318-322.

[5] R. A. Chevalier, Hydrodynamic models of supernova explosions, Fundamentals of Cosmic Physics 7 (1981), 1-58.

[6] U. C. De, K. Matsumoto, and A. A. Shaikh, On Lorentzian para-Sasakian manifolds, Rendiconti del Seminario Mat. de Messina, al n. 3 (1999), 149-158.

[7] F. Defever, R. Deszcz, M. Hotloś, M. Kucharski, and Z. Sentürk, Generalisations of Robertson-Walker spaces, Annales Univ. Sci. Budapest. Eötvös Sect. Math. 43 (2000), $13-24$.

[8] J. Deprez, W. Roter, and L. Verstraelen, Conditions on the projective curvature tensor of conformally flat Riemannian manifolds, Kyungpook Math. J. 29 (1989), no. 2, 153165.

[9] R. Deszcz, On pseudosymmetric spaces, Bull. Soc. Math. Belg. Ser. A 44 (1992), no. 1, $1-34$.

[10] R. Deszcz, F. Dillen, L. Verstraelen, and L. Vrancken, Quasi-Einstein totally real submanifolds of the nearly Kähler 66-sphere, Tohoku Math. J. (2) 51 (1999), no. 4, 461-478.

[11] R. Deszcz, M. Glogowska, M. Hotloś, and Z. Sentürk, On certain quasi-Einstein semisymmetric hypersurfaces, Annales Univ. Sci. Budapest. Eötvös Sect. Math. 41 (1998), $151-164$.

[12] R. Deszcz, M. Hotloś, and Z. Sentürk, Quasi-Einstein hypersurfaces in semi-Riemannian space forms, Colloq. Math. 81 (2001), no. 1, 81-97.

[13] _ On curvature properties of quasi-Einstein hypersurfaces in semi-Euclidean spaces, Soochow J. Math. 27 (2001), no. 4, 375-389.

[14] R. Deszcz, P. Verheyen, and L. Verstraelen, On some generalized Einstein metric conditions,Publ. Inst. Math. (Beograd) (N.S.) 60(74) (1996), 108-120.

[15] R. Deszcz, L. Verstraelen, and S. Yaprak, Warped products realizing a certain condition of pseudosymmetry type imposed on the Weyl curvature tensor, Chinese J. Math. 22 (1994), no. 2, 139-157.

[16] , Pseudosymmetric hypersurfaces in 4-dimensional spaces of constant curvature, Bull. Inst. Math. Acad. Sinica 22 (1994), no. 2, 167-179.

[17] R. Deszcz and M. Hotloś, On some pseudosymmetry type curvature condition, Tsukuba J. Math. 27 (2003), no. 1, 13-30.

[18] _ On hypersurfaces with type number two in space forms, Ann. Univ. Sci. Budapest. Eötvös Sect. Math. 46 (2003), 19-34.

[19] R. Deszcz and M. Glogowska, Examples of nonsemisymmetric Ricci-semisymmetric hypersurfaces, Colloq. Math. 94 (2002), no. 1, 87-101.

[20] D. Ferus, A Remark on Codazzi Tensors on Constant Curvature Space, Lecture Notes Math. 838, Global Differential Geometry and Global Analysis, Springer-Verlag, New York, 1981.

[21] M. Glogowska, Semi-Riemannian manifolds whose weyl tensor is a Kulkarni-Nomizu square, Publ. Inst. Math. (Beograd), Tome 72 (2002), no. 86, 95-106.

[22] _ On quasi-Einstein Cartan type hypersurfaces, J. Geom. Phys. 58 (2008), no. 5, $599-614$

[23] F. Gouli-Andreou and E. Moutafi, Two classes of pseudosymmetric contact metric 3manifolds, Pacific J. Math. 239 (2009), no. 1, 17-37. 
[24] T. Ikawa and M. Erdogan, Sasakian manifolds with Lorentzian metric, Kyungpook Math. J. 35 (1996), no. 3, 517-526.

[25] H. Karchar, Infinitesimal characterization of Friedmann Universe, Arch. Math. Basel 38 (1992), 58-64.

[26] K. Matsumoto, On Lorentzian paracontact manifolds, Bull. Yamagata Univ. Natur. Sci. 12 (1989), no. 2, 151-156.

[27] K. Matsumoto and I. Mihai, On a certain transformation in a Lorentzian para-Sasakian manifold, Tensor N. S. 47 (1988), no. 2, 189-197.

[28] I. Mihai and R. Rosca, On Lorentzian P-Sasakian manifolds, Classical analysis (Kazimierz Dolny, 1991), 155-169, World Sci. Publ., River Edge, NJ, 1992.

[29] I. Mihai, A. A. Shaikh, and U. C. De, On Lorentzian para-Sasakian manifolds, Korean J. Math. Sciences 6 (1999), 1-13.

[30] M. Novello and M. J. Reboucas, The stability of a rotating universe, The Astrophysics J. 225 (1978), 719-724.

[31] B. O'Neill, Semi Riemannian Geometry with Applications to Relativity, Academic Press, Inc., 1983.

[32] P. J. E. Peebles, The Large-Scale Structure of the Universe, Princeton Univ. Press, 1980.

[33] J. A. Schouten, Ricci Calculus, (2nd Ed.), Springer-Verlag, Berlin, 1954.

[34] A. A. Shaikh, On Lorentzian almost paracontact manifolds with a structure of the concircular type, Kyungpook Math. J. 43 (2003), no. 2, 305-314.

[35] A. A. Shaikh, T. Basu, and K. K. Baishya, On the existence of locally $\phi$-recurrent LP-Sasakian manifold, Bull. Allahabad Math. Soc. 24 (2009), no. 2, 281-295.

[36] A. A. Shaikh and K. K. Baishya, On $\phi$-symmetric LP-Sasakian manifolds, Yokohama Math. J. 52 (2006), no. 2, 97-112.

[37] - Some results on LP-Sasakian manifolds, Bull. Math. Soc. Sc. Math. Roumanie Tome, 49(97) (2006), no. 2, 193-205.

[38] _ On concircular structure spacetimes, J. Math. Stat. 1 (2005), no. 2, 129-132.

[39] _ On concircular structure spacetimes II, Amer. J. Appl. Sci. 3 (2006), 1790-1794.

[40] A. A. Shaikh and S. Biswas, On LP-Sasakian manifolds, Bull Malaysian Math. Sci. Soc. 27 (2004), no. 1, 17-26.

[41] M. Spivak, A Comprehensive Introduction to Differential Geometry, Publish and Perish, Vol. I, 1970.

[42] T. Takahashi, Sasakian $\phi$-symmetric spaces, Tohoku Math. J. 29 (1977), no. 1, 91-113.

\author{
Absos Ali Shaikh \\ Department of Mathematics \\ UNIVERSITY OF BURDWAN \\ BURDWAN - 713104 \\ West BEnGAL, INDIA \\ E-mail address: aask2003@yahoo.co.in \\ YOUNG HO KIM \\ Department of Mathematics \\ KYUnGPOOK NATIONAL UNIVERSITY \\ TAEGU 702-701, KoreA \\ E-mail address: yhkim@knu.ac.kr \\ Shyamal Kumar Hui \\ Department of Mathematics \\ UNIVERSITY OF BURDWAN \\ BURDWAN - 713104 \\ West Bengal, India
}

